\title{
Candidemia in a Pediatric Population: A 10-year Indian Study
}

\author{
Ranjeeta Adhikary ${ }^{1}$, Bhavana Malavalli Venkatesh ${ }^{2}$, Sangeeta Joshi ${ }^{3}$, Anjana Aravinda $^{4}$
}

\begin{abstract}
Introduction: Candidemia has emerged as one of the life-threatening causes of invasive infection in both adults and children worldwide. Materials and methods: We performed a retrospective study of children ( $\leq 16$ years) with candidemia diagnosed in our center in 2010 to 2019. Demographics, comorbidities, Candida species distribution, antifungal susceptibility, and outcomes were analyzed.

Results: A total of 96 children were identified in the last 10 years. The predominant species identified were C. tropicalis (23\%), C. parapsilosis (15.6\%), C. pelliculosa (15.6\%), C. albicans (13.6\%), C. krusei (7.3\%), and C. haemulonii (5.2\%). Male to female ratio was 2:1. The most common risk factor was found to be congenital malformations (27\%), followed by hematological malignancy in $13.5 \%$. Candidemia was diagnosed while being admitted in the intensive care unit in $74 \%, 14.5 \%$ in wards, and $11.5 \%$ in outpatients. The overall mortality rate was found to be $31.3 \%$. C. tropicalis was found to be sensitive to fluconazole in $95.5 \%$, flucytocine in $95.2 \%$, and $100 \%$ susceptible to amphotericin B, voriconazole, and caspofungin.

Conclusion: Invasive candidiasis occurs frequently in hospitalized patients and is associated with high mortality rates. C. tropicalis was the most frequently isolated species. We have observed a shift in Candida spp. with an increasing isolation of C. pelliculosa. The occurrence of azole resistance is a matter of concern.

Clinical significance: This type of data analysis is needed to track trends of serious infection and to develop guidelines for infection control strategies and antimicrobial stewardship program.

Keywords: Candidemia, Children, Invasive candidiasis.

Pediatric Infectious Disease (2021): 10.5005/jp-journals-10081-1255
\end{abstract}

\section{INTRODUCTION}

The yeast belonging to the genus Candida forms a part of the normal skin and mucosal flora in humans. It is capable of causing invasive candidiasis which includes, among other clinical manifestations, intra-abdominal infections, osteomyelitis, and bloodstream infections (candidemia). Candidemia being the most common type of invasive candidiasis remains a serious cause of illness with fatal outcome globally. ${ }^{1}$ Data from the National Healthcare Safety Network have identified Candida species as the fifth most common cause of healthcare infection in hospitalized US patients behind coagulase-negative Staphylococci. ${ }^{2}$ In a review article, the fungi have been documented as the second most common pathogen isolated in hospitalized septic children and found to be the leading cause of death in cancer children or following transplant, mostly in an organ or hematopoietic stem cell transplant. ${ }^{3}$

There are also reports suggesting an increase in candidemia. ${ }^{4-6}$ Advances in medical and surgical management have attributed to an increase in invasive candidiasis. Other associated risk factors include severe trauma, long-term use of antibiotics, major surgical procedures, mucosal colonization by Candida spp., indwelling vascular catheters or prosthetic devices, total parenteral nutrition, immunosuppressive therapy, premature infants, and use of mechanical ventilator support. ${ }^{1,7-10}$

Candidemias are reported worldwide in all age-groups, especially among critically ill, immunocompromised patients or those with complicated medical condition. ${ }^{11}$ It is often associated with prolonged hospitalizations, high healthcare costs, substantial morbidity, and all-cause in-hospital mortality of up to $30 \%{ }^{1}$

There are more than 17 species of Candida causing invasive candidiasis in humans. More than $90 \%$ of these infections are due

\author{
1,2,4 Department of Laboratory Medicine-Microbiology, Manipal \\ Hospital, Bengaluru, Karnataka, India \\ ${ }^{3}$ Department of Microbiology, Manipal Hospital Dwarka, Delhi, \\ India
}

Corresponding Author: Ranjeeta Adhikary, Department of Laboratory Medicine-Microbiology, Manipal Hospital, Bengaluru, Karnataka, India, Phone: +91 8025023355, e-mail: adhikary.ranjeeta@gmail.com

How to cite this article: Adhikary R, Venkatesh BM, Joshi S, et al. Candidemia in a Pediatric Population: A 10-year Indian Study. Pediatr Inf Dis 2021;3(1):1-8.

Source of support: Nil

Conflict of interest: None

to five species: C. albicans, C. glabrata, C. parapsilosis, C. tropicalis, and $C$. krusei. The list of reported species will continue to grow with modern methods of identification. ${ }^{6,12,13}$

C. albicans is the most common species causing candidiasis in the United States. ${ }^{1}$ However, significant geographical differences in species distribution have been observed. ${ }^{6,14} \mathrm{~A}$ shift in the epidemiology of candidemia from C. albicans toward non-albicans Candida spp. has been a global concern in recent times. $6,9,10,14,15$ There has been an increase in the proportion of infections due to C. glabrata and C. parapsilosis in the last few decades. These species show higher levels of resistance to antifungal agents and therefore might be associated with higher mortality than C. albicans. ${ }^{1,9}$ Equally concerning is $C$. auris, a multidrug resistant, emerging agent of candidemia. It was first described in 2009 and has since been reported in 30 countries, including India. ${ }^{1,16}$ Understanding the epidemiology of Candida species is critical, as clinicians often begin 
empiric therapy before antifungal susceptibilities are reported from the isolate.

\section{Materials and Methods}

A retrospective analysis of all cases of candidemia in children was carried out from January 1, 2010, to December 31, 2019, in a single 600 -bed tertiary-care hospital in southern India. Candidemia was defined as an isolation of Candida species from one or more blood cultures in a patient with supportive clinical correlation.

All candidemia were identified through the microbiological laboratory database. Data regarding demographic characteristics and clinical risk factors were collected from the hospital information system. All children were categorized into four age-groups: neonate (birth to $<1$ month), infant ( 1 month to $<2$ years), children ( 2 to $<12$ years), and adolescent (12-16 years).

Blood samples were collected under aseptic condition in BacT/ ALERT (bioMerieux) culture bottles for aerobic bacterial and fungal growth and incubated at $37^{\circ} \mathrm{C}$ for 7 days. When BactAlert indicated positive, bottles were unloaded and preliminary Gram stain and subcultures were made on 5\% sheep blood agar and MacConkey agar. The isolates were further identified into species level by (a) germ tube production, (b) pigmentation on chromogenic medium (CHROM agar Candida), and (c) carbohydrate assimilation tests using the VITEK 2 compact system (bioMerieux). Adequate quality control measures were undertaken with reference strains $C$. krusei ATCC 6258 and C. parapsilosis ATCC 22019.

Antifungal susceptibility was also done for fluconazole, amphotericin B, voriconazole, flucytosine, caspofungin, and micafungin in VITEK 2 compact system. The interpretative minimum inhibitory concentration (MIC) breakpoints of the tested antifungals (fluconazole, voriconazole, caspofungin, and micafungin) were those suggested by the CLSI M27-A3 document, and for amphotericin B it was based on EUCAST guidelines. ${ }^{17,18}$ For flucytosine, the categorical result was obtained according to the breakpoints provided by the VITEK-2 system. ${ }^{19}$

\section{Results}

There were 123 different episodes of candidemia detected in 96 children during the 10-year study period. Eighteen patients had more than one episode of candidemia. One patient had two different Candida species in a single episode. Only the first isolate of the patient (total 96 isolates) was taken into consideration for the study. Most of the blood isolates have been caused by non- $C$. albicans (86.4\%). Overall, C. tropicalis accounted for $23 \%$ of the isolates, followed by C. parapsilosis and C. pelliculosa at $15.6 \%$, C. albicans at $13.6 \%$, C. krusei at 7.3\%, C. haemulonii at $5.2 \%, C$. lipolytica and C. famata at $3.1 \%$, and relatively rare were C. glabrata, C. guilliermondii, C. Iusitaniae, and C. utilis at 2.1\%. Five isolates (5.2\%) of Candida were not identified to species.

Overall, a total of 55 (57.3\%) isolates were seen in 2010 to 2014, and 41 (42.7\%) isolates in 2015 to 2019. Three new species, C. famata, C. glabrata, and C. utilis were seen in 2015 to 2019. The increased isolation of C. pelliculosa from 5 isolates in 2010 to 2014 to 10 isolates in 2015 to 2019 was found to be statistically significant with a $p$ value of 0.04. C. famata was also found to be statistically significant with a $p$ value of 0.04 , as 3 cases were reported in 2015-2019 and nil in 2010-2014. The Chi-square test was used for analysis of trends across years. In 2010 to 2014, C. tropicalis was the most common isolate, followed by C. albicans, and C. parapsilosis. But in 2015 to 2019, C. pelliculosa was the most common isolate followed by C. tropicalis and C. parapsilosis. Table 1 shows species distribution of 96 Candida bloodstream isolates in 2 groups of 5 years, 2010 to 2014 and 2015 to 2019.

We have isolated $74 \%$ of the isolates from ICU, $14.5 \%$ from inpatients, and $11.5 \%$ from outpatients. It is to be noted that C. pelliculosa $(n=15), C$. lusitaniae $(n=2)$, and C. utilis $(n=2)$ were isolated from patients admitted in the ICU. In contrast, C. guilliermondii $(n=2)$ was found in patients admitted in the ward as summarized in Table 2.

We identified 31 (32.3\%) neonates, 42 (43.8\%) infants, 18 (18.8\%) children, and 5 (5.2\%) adolescents with Candida bloodstream infection (BSI). Therefore, $76 \%$ of the isolates were seen from birth up to $<2$ years. Both C. tropicalis (22.6\%) and C. pelliculosa $(22.6 \%)$ were the predominant species isolated in neonates; $C$. famata, C. glabrata, and C. utilis were not found in in this age-group. But in the infants, C. tropicalis (23.8\%) followed by C. albicans (16.7\%) were isolated more frequently. Among the children, C. parapsilosis (33.3\%) followed by $C$. tropicalis (16.7\%) and in adolescents; $C$. tropicalis (40\%) was most frequently isolated. Table 3 shows distribution of Candida species according to age-group.

The demographic study of the 96 candidemia patients showed that the median age was 2 years (range, 7 days to $\leq 16$ years) with $66.7 \%$ males and $33.3 \%$ females. In relation to clinical characteristics, $27.1 \%$ of candidemia occurred in children with congenital malformation, followed by hematological malignancy in $13.5 \%$. Other predisposing factors are lung disease $(9.4 \%)$, prematurity $(8.3 \%)$, surgery $(7.3 \%)$, septic shock $(6.3 \%)$, neonatal sepsis (6.3\%), low birth weight (4.2\%); low APGAR score, and other medical condition (3.1\%); cardiovascular disease, hemodialysis, renal failure, trauma, IUGR, fever seizure, and respiratory distress each at $2.1 \%$; and diabetes mellitus and solid malignancy each at $1 \%$. The demographic details and clinical characteristics of the patients are summarized in Table 4.

The overall mortality in our study was found to be $31.3 \%$ (30/96). The Candida species included were C. pelliculosa (7,

Table 1: Species distribution of 96 Candida bloodstream isolates in southern India, 2010-2019

\begin{tabular}{|c|c|c|c|c|}
\hline \multirow[b]{2}{*}{ Species } & \multicolumn{3}{|c|}{ No. (\%) of isolates } & \multirow[b]{2}{*}{$p^{a}$} \\
\hline & 2010-2014 & 2015-2019 & Total & \\
\hline C. tropicalis & $14(25.4)$ & $8(19.5)$ & $22(23)$ & 0.49 \\
\hline C. parapsilosis & $8(14.5)$ & $7(17)$ & 15 (15.6) & 0.74 \\
\hline C. pelliculosa & $5(9.1)$ & $10(24.3)$ & 15 (15.6) & 0.04 \\
\hline C. albicans & $10(18.2)$ & $3(7.3)$ & $13(13.6)$ & 0.12 \\
\hline C. krusei & $5(9.1)$ & $2(5)$ & $7(7.3)$ & 0.43 \\
\hline $\begin{array}{l}\text { Candida. species } \\
\text { non-albicans }\end{array}$ & $4(7.3)$ & $1(2.4)$ & $5(5.2)$ & 0.29 \\
\hline C. haemulonii & $3(5.4)$ & $2(5)$ & $5(5.2)$ & 0.90 \\
\hline C. lipolytica & $3(5.4)$ & 0 & $3(3.1)$ & 0.13 \\
\hline C. famata & 0 & $3(7.3)$ & $3(3.1)$ & 0.04 \\
\hline C. glabrata & 0 & $2(5)$ & $2(2.1)$ & 0.10 \\
\hline C. guilliermondii & $1(1.8)$ & $1(2.4)$ & $2(2.1)$ & 0.83 \\
\hline C. Iusitaniae & $2(3.6)$ & 0 & $2(2.1)$ & 0.22 \\
\hline C. utilis & 0 & $2(5)$ & $2(2.1)$ & 0.10 \\
\hline Total & $55(57.3 \%)$ & $41(42.7 \%)$ & 96 & \\
\hline
\end{tabular}

${ }^{\mathrm{a}}$ Chi-squared test for trend 
23.3\%), C. tropicalis (6, 20\%), C. parapsilosis (5, 16.7\%), C. albicans (3, $10 \%)$, C. haemulonii (3, 10\%), C. krusei (3,10\%), C. famata (1, 3.3\%), C. guilliermondii (1,3.3\%), Candida spp. non albicans (1, 3.3\%). All the children with C. lipolytica (3), C. glabrata (2), C. Iusitaniae (2), and C. utilis (2) recovered from candidemia. Table 5 shows number of died patients in each category of Candida spp.

Out of a total number of 71 intensive care unit (ICU) patients, 14 inpatients and 11 outpatients, 24 (33.8\%), 4 (28.6\%), and 2 (18.2\%), respectively, in each group had a fatal outcome. Six patients from ICU were discharged on request and were lost to follow-up. The mortality was found to be $29 \%$ among the neonates, $23.8 \%$ among the infant, 39\% among the children, and $80 \%$ among the adolescent age-groups. This was found to be statistically significant in the age group of birth to $<2$ years when compared 2 to 16 years ( $p$ value $=$ 0.04). Location and age-wise distribution of the expired candidemia cases are summarized in Table 6.

Table 2: Species distribution and location of 96 Candida species bloodstream infection

\begin{tabular}{lllll}
\hline & $\begin{array}{l}\text { Total no. (\%) } \\
\text { of isolates } \\
\text { Species }\end{array}$ & $\begin{array}{l}\text { ICU total } \\
\text { no. (\%) }\end{array}$ & $\begin{array}{l}\text { IP total } \\
\text { no. }(\%)\end{array}$ & $\begin{array}{l}\text { OP total } \\
\text { no. }(\%)\end{array}$ \\
\hline C. tropicalis & $22(23)$ & $18(25.3)$ & $2(14.3)$ & $2(18.2)$ \\
C. parapsilosis & $15(15.6)$ & $8(11.3)$ & $3(21.4)$ & $4(36.4)$ \\
C. pelliculosa & $15(15.6)$ & $15(22.1)$ & 0 & 0 \\
C. albicans & $13(13.6)$ & $10(14)$ & $1(7.1)$ & $2(18.2)$ \\
C. krusei & $7(7.3)$ & $5(7)$ & & $2(18.2)$ \\
Candida. species & $5(5.2)$ & $2(2.8)$ & $2(14.3)$ & $1(9.1)$ \\
non-albicans & & & & \\
C. haemulonii & $5(5.2)$ & $4(5.6)$ & $1(7.1)$ & 0 \\
C. lipolytica & $3(3.1)$ & $3(4.2)$ & 0 & 0 \\
C. famata & $3(3.1)$ & $1(1.4)$ & $2(14.3)$ & 0 \\
C. glabrata & $2(2.1)$ & $1(1.4)$ & $1(7.1)$ & 0 \\
C. guilliermondii & $2(2.1)$ & 0 & $2(14.3)$ & 0 \\
C. lusitaniae & $2(2.1)$ & $2(2.8)$ & 0 & 0 \\
C. utilis & $2(2.1)$ & $2(2.8)$ & 0 & 0 \\
Total & 96 & $71(74)$ & $14(14.5)$ & $11(11.5)$ \\
\hline
\end{tabular}

Among the 96 isolates, 94 isolates were available for the susceptibilities of antifungal agents. For all 94 Candida BSI isolates combined, the activity of each agent $(\mu \mathrm{g} / \mathrm{mL})$, expressed as the $\mathrm{MIC}_{50} / \mathrm{MIC}_{90}$ (and the percentage of susceptible isolates), was as follows: fluconazole, $1 / 8$ (71\%); amphotericin B, 0.5/1 (91.5\%); voriconazole, $\leq 0.12 / 0.25$ (99\%); flucytosine, $\leq 1 / 8(87.8 \%)$; caspofungin, $\leq .25 / 0.5$ (94\%); and micafungin, 0.5/0.12 (100\%). C. tropicalis, the most commonly isolated species, was $95.5 \%$ sensitive to fluconazole, $95.2 \%$ sensitive to flucytosine, and $100 \%$ sensitive to amphotericin B, voriconazole, caspofungin, and micafungin. The isolates in which no interpretable breakpoints exist in CLSI/EUCAST, the MIC values and the range of the measured MICs are given, and no statement concerning the clinical sensitivity could be made. All seven C. krusei isolates are considered to be resistant to fluconazole, irrespective of their MIC. The in vitro antifungal susceptibilities as determined by VITEK 2 system is summarized in Table 7.

\section{Discussion}

Significant geographic variation among BSI due to Candida spp. is observed in different parts of the world. In our study in pediatric age-group, BSIs due to non-Candida albicans spp. were more common $(86.4 \%)$ than C. albicans (13.6\%). This finding is consistent with other studies from Turkey, Saudi Arabia, India, and Mexico. ${ }^{7,10,20-23}$ An important finding from an Indian study was the emergence of Candida spp. as the second most common cause of BSI in pediatric ICUs (PICU) after coagulase-negative Staphylococcus, instead of gram-negative bacteria. ${ }^{20}$ In our study, the top five isolates were C. tropicalis (23\%), C. parapsilosis (15.6\%), C. pelliculosa (15.6\%), C. albicans (13.6), and C. krusei (7.3\%). In a study from North India, C. tropicalis (39\%) was the commonest isolate recovered, followed by C. parapsilosis (18\%), C. albicans (12\%), C. glabrata (12\%), C. kefyr (9\%), C. pelliculosa (5\%), and C. krusei (5\%). ${ }^{21}$ In another recent study from North India, C. tropicalis (38.2\%) was the most common Candida spp., followed by C. pelliculosa (16.4\%), and C. albicans (12.7\%). ${ }^{20}$ In our study, we have not reported C. kefyr, although our percentage isolation of C. pelliculosa and C. albicans was similar. In contrast, in a study from South India, C. parapsilosis was the predominant pathogen among children. ${ }^{24}$

Table 3: Distribution of Candidemia in southern India, 2010 to 2019, according to species, and age-group

\begin{tabular}{|c|c|c|c|c|c|}
\hline \multirow[b]{2}{*}{ Species } & \multicolumn{5}{|c|}{ No. (\%) of isolates in each age group } \\
\hline & $\begin{array}{l}\text { Neonate (birth to }<1 \\
\text { month) }\end{array}$ & $\begin{array}{l}\text { Infant ( } 1 \text { month to }<2 \\
\text { years) }\end{array}$ & $\begin{array}{l}\text { Children }(2 \text { to }<12 \\
\text { years) }\end{array}$ & $\begin{array}{l}\text { Adolescent (12-16 } \\
\text { years) }\end{array}$ & Total no. (\%) \\
\hline C. tropicalis & $7(22.6)$ & $10(23.8)$ & $3(16.7)$ & $2(40 \%)$ & $22(23)$ \\
\hline C. parapsilosis & $2(6.4)$ & $6(14.3)$ & $6(33.3)$ & $1(20 \%)$ & $15(15.6)$ \\
\hline C. pelliculosa & $7(22.6)$ & $6(14.3)$ & $2(11.1)$ & 0 & $15(15.6)$ \\
\hline C. albicans & $5(16.1)$ & $7(16.7)$ & $1(5.5)$ & 0 & $13(13.6)$ \\
\hline C. krusei & $4(12.9)$ & $3(7.14)$ & 0 & 0 & $7(7.3)$ \\
\hline C. species non-albicans spp. & 0 & $3(7.14)$ & $1(5.5)$ & $1(20 \%)$ & $5(5.2)$ \\
\hline C. haemulonii & $2(6.4)$ & $2(4.8)$ & 0 & $1(20 \%)$ & $5(5.2)$ \\
\hline C. lipolytica & $3(9.8)$ & 0 & 0 & 0 & $3(3.1)$ \\
\hline C. famata & 0 & $1(2.4)$ & $2(11.1)$ & 0 & $3(3.1)$ \\
\hline C. glabrata & 0 & $1(2.4)$ & $1(5.5)$ & 0 & $2(2.1)$ \\
\hline C. guilliermondii & 0 & $1(2.4)$ & $1(5.5)$ & 0 & $2(2.1)$ \\
\hline C. Iusitaniae & $1(3.2)$ & $1(2.4)$ & 0 & 0 & $2(2.1)$ \\
\hline C. utilis & 0 & $1(2.4)$ & $1(5.5)$ & 0 & $2(2.1)$ \\
\hline Total & $31(32.3)$ & $42(43.8)$ & $18(18.8)$ & 5 (5.2\%) & 96 \\
\hline
\end{tabular}


Table 4: Demographics and clinical characteristics of 96 patients with Candidemia

\begin{tabular}{ll}
\hline Characteristics & No. (\%) \\
\hline Male & $64(66.7)$ \\
Female & $32(33.3)$ \\
Congenital malformations & $26(27)$ \\
Hematological malignancy & $13(13.5)$ \\
Lung disease & $9(9.4)$ \\
Prematurity & $8(8.3)$ \\
Surgery & $7(7.3)$ \\
Septic shock & $6(6.3)$ \\
Neonatal sepsis & $6(6.3)$ \\
Low birth weight & $4(4.2)$ \\
Low APGAR score & $3(3.1)$ \\
Other medical condition & $3(3.1)$ \\
Cardiovascular disease & $2(2.1)$ \\
Hemodialysis & $2(2.1)$ \\
Renal failure & $2(2.1)$ \\
Trauma & $2(2.1)$ \\
IUGR & $2(2.1)$ \\
Fever seizure & $2(2.1)$ \\
Respiratory distress & $2(2.1)$ \\
Diabetes mellitus & $1(1)$ \\
Solid malignancy & $1(1)$ \\
\hline
\end{tabular}

Table 5: Number of expired patients in each category of Candida spp

\begin{tabular}{lll}
\hline Species & Total no. of isolates & Total no. expired (\%) \\
\hline C. pelliculosa & 15 & $7(23.3)$ \\
C. tropicalis & 22 & $6(20)$ \\
C. parapsilosis & 15 & $5(16.7)$ \\
C. albicans & 13 & $3(10)$ \\
C. haemulonii & 5 & $3(10)$ \\
C. famata & 3 & $1(3.3)$ \\
C. species non-albicans & 5 & $1(3.3)$ \\
spp. & & \\
C. guilliermondii & 2 & $1(3.3)$ \\
C. krusei & 7 & 0 \\
C. lipolytica & 3 & 0 \\
C. glabrata & 2 & 0 \\
C. lusitaniae & 2 & 0 \\
C. utilis & 2 & 0 \\
Total no (\%) & 96 & $30(31.3)$ \\
\hline
\end{tabular}

Table 6: Number of expired patients of Candidemia in each location and age-group

\begin{tabular}{lll}
\hline & Total no. of isolates & $\begin{array}{l}\text { No. of expired patients } \\
\text { (\%) }\end{array}$ \\
\hline ICU & 71 & $24(33.8)$ \\
IP & 14 & $4(28.6)$ \\
OP & 11 & $2(18.2)$ \\
Neonate & 31 & $9(29)$ \\
Infant & 42 & $10(23.8)$ \\
Children & 18 & $7(39)$ \\
Adolescent & 5 & $4(80)$ \\
\hline
\end{tabular}

We found statistically significant trend overtime regarding the distributions of $C$. pelliculosa and C. famata. In a review article comparing the overall distribution of isolates recovered from US vs non-US study sites, a difference was observed in the proportions of Candida species. Although it was observed that $C$. albicans was the most common species isolated overall, it accounted for a lower percentage of all isolates in non-US sites when compared to US sites. In contrast, C. guilliermondii was recovered more frequently in non-US study sites, while $C$. krusei was isolated in larger proportion in US than in non-US study sites. ${ }^{3}$ C. krusei in our study was $7.3 \%$.

A rarely encountered Candida species that has received worldwide attention as causing invasive infections is $C$. auris. This is commonly misidentified as $C$. haemulonii by commercial methods of clinical diagnosis. ${ }^{16,25}$ Although we have not reported C. auris in our study, the overall C. haemulonii isolation was $5.2 \%$ in the pediatric age-group.

We identified low prevalence of C. lipolytica (3.1\%), C. famata (3.1\%), C. glabrata (2.1\%), C. guilliermondii (2.1\%), C. Iusitaniae (2.1\%), and C. utilis (2.1\%) in our study as seen in other settings and geographical regions. ${ }^{14,26-28}$ Moreover, although C. glabrata remained relatively uncommon in our study $(2.1 \%)$, the proportion of invasive Candida infections caused by C. pelliculosa increased from $9.1 \%$ in 2010 to 2014 to $24.3 \%$ in 2015 to 2019. C. pelliculosa is considered a rare non-C. albicans spp. pathogen. Despite being uncommon, the role of this pathogen causing outbreaks has been reported in children. ${ }^{4,15,29,30}$ Similarly, C. glabrata in another study has reported increase from $2.6 \%$ in 2003 to $23.7 \%$ in $2012 .{ }^{12}$

There were significant differences in Candida spp. among different locations in the healthcare setting. Majority of the isolates (74\%) are from ICU, with C. tropicalis (18/22, 81.8\%), C. pelliculosa $(15 / 15,100 \%)$, and C. albicans $(10 / 13,76.9 \%)$ as the most frequently isolated Candida species. It is noteworthy that uncommon Candida species, C. pelliculosa; C. lipolytica, C. lusitaniae, and C. utilis, were only isolated from ICU except $C$. guilliermondii.

Significant differences in Candida spp. were observed among different age-groups in children. In our study, we have demonstrated both $C$. tropicalis and C. pelliculosa (22.6\%) as the predominant species in the neonates, whereas $C$. albicans was only $16.1 \%$ among the neonates compared to $13.6 \%$ in the overall pediatric group. Among the infants, we found C. tropicalis (23.8\%) followed by C. albicans (16.7\%). Candidemia was infrequent in adolescents with 5 cases among 96 patients. In a study from Chile, neonates had the highest proportion of cases of C. albicans $(60 \%)$ compared to $37.3 \%$ in the overall pediatric group. ${ }^{31}$ In a review article by Warris A, it has been mentioned that the vast majority of the infections caused by Candida species in the pediatric population were due to C. albicans or C. parapsilosis and C. krusei was less prevalent. ${ }^{25}$ Similar finding was seen in a nationwide surveillance study in Mexico. ${ }^{23}$

The median age was 2 years (range, 7 days to $\leq 16$ years), whereas the median patient age in another study was 50 months (range, 6 months to $\leq 18$ years). ${ }^{32}$ Candidemia was seen to be more prevalent in male with $66.7 \%$ of infected children than female with $33.3 \%$. It corroborates with another study on pediatric candidemia where $55.4 \%$ patients infected were males and $44.6 \%$ were female. ${ }^{32}$ However, this observation is not much in agreement with other studies reporting female predominance. ${ }^{21}$

In a study among the European pediatric population, cancer/ allogeneic hematopoietic stem cell transplantation (43\%) and congenital malformations/syndromes (21\%) were the predominant 
Candidemia in a Pediatric Population: A 10-year Indian Study

Table 7: In vitro antifungal susceptibilities of 94 isolates of Candida species to fluconazole, amphotericin B, voriconazole, flucytosine, caspofungin, and micafungin as determined by using the VITEK-2 system

\begin{tabular}{|c|c|c|c|c|c|}
\hline Species (no. of isolates) & $\begin{array}{l}\text { Agents (no. available } \\
\text { for susceptibilities) }\end{array}$ & MIC $(\mu g / m L)$ range & $M I C_{50}(\mu \mathrm{g} / \mathrm{mL})$ & $M I C_{90}(\mu \mathrm{g} / \mathrm{mL})$ & Percentage sensitive \\
\hline \multirow[t]{6}{*}{ C. tropicalis (22) } & FLU (22) & $\leq 1-16$ & $\leq 1$ & 2 & 95.5 \\
\hline & $\mathrm{AMB}(22)$ & $\leq 0.25-1$ & 0.5 & 0.5 & 100 \\
\hline & VOR (22) & $\leq 0.12-1$ & $\leq 0.12$ & $\leq 0.12$ & 100 \\
\hline & FC (22) & $\leq 1-32$ & $\leq 1$ & $\leq 1$ & 95.2 \\
\hline & CAS (12) & $\leq 0.12-0.25$ & 0.25 & 0.25 & 100 \\
\hline & MIC (8) & $\leq 0.06$ & $\leq 0.06$ & $\leq 0.06$ & 100 \\
\hline \multirow[t]{6}{*}{ C. parapsilosis (15) } & FLU (15) & $\leq 0.5-2$ & 1 & 2 & 100 \\
\hline & $\mathrm{AMB}(15)$ & $\leq 0.25-1$ & 0.5 & 0.5 & 100 \\
\hline & VOR (15) & $\leq 0.12$ & $\leq 0.12$ & $\leq 0.12$ & 100 \\
\hline & FC (15) & $\leq 1$ & $\leq 1$ & $\leq 1$ & 100 \\
\hline & CAS (12) & $\leq 0.12-1$ & 0.25 & 1 & 100 \\
\hline & MIC (8) & $0.25-0.5$ & 0.5 & 0.5 & 100 \\
\hline \multirow[t]{6}{*}{ C. pelliculosa (15) } & FLU (15) & $\leq 1-8$ & 2 & 4 & 66.7 \\
\hline & $\mathrm{AMB}(15)$ & $\leq 0.25-0.5$ & 0.5 & 0.5 & \\
\hline & VOR (15) & $\leq 0.12-0.25$ & $\leq 0.12$ & 0.25 & \\
\hline & FC (15) & $\leq 1$ & $\leq 1$ & $\leq 1$ & 100 \\
\hline & CAS (11) & $\leq 0.12-0.25$ & 0.25 & 0.25 & \\
\hline & MIC (10) & $\leq 0.06-0.12$ & $\leq 0.06$ & 0.12 & \\
\hline \multirow[t]{6}{*}{ C. albicans (13) } & FLU (13) & $\leq 1-8$ & $\leq 1$ & 2 & 100 \\
\hline & $\mathrm{AMB}(13)$ & $\leq 0.25-1$ & 1 & 1 & 100 \\
\hline & VOR (13) & $\leq 0.12$ & $\leq 0.12$ & $\leq 0.12$ & 100 \\
\hline & FC (13) & $\leq 1-\geq 64$ & $\leq 1$ & $\geq 64$ & 92.3 \\
\hline & CAS (3) & $\leq 0.12$ & $\leq 0.12$ & $\leq 0.12$ & 100 \\
\hline & MIC (3) & $\leq 0.06$ & $\leq 0.06$ & $\leq 0.06$ & 100 \\
\hline \multirow[t]{6}{*}{ C. krusei $(n=7)$} & FLU (7) & $\geq 4-64$ & 8 & $\geq 64$ & Intrinsically resistant \\
\hline & $\mathrm{AMB}(7)$ & $\leq 0.25-4$ & 1 & 4 & 71.4 \\
\hline & $\operatorname{VOR}(7)$ & $\leq 0.12-0.5$ & $\leq 0.12$ & 0.5 & 100 \\
\hline & $\mathrm{FC} \mathrm{(7)}$ & $4-16$ & 8 & 16 & 14.3 \\
\hline & CAS (4) & $\leq 0.12-0.5$ & 0.25 & 0.5 & 75 \\
\hline & MIC (2) & 0.12 & 0.12 & 0.12 & 100 \\
\hline \multirow[t]{6}{*}{ C. haemulonii $(n=5)$} & FLU (5) & $16-32$ & 16 & 32 & 0 \\
\hline & $\mathrm{AMB}(5)$ & $2-\geq 16$ & 8 & $\geq 16$ & \\
\hline & VOR (5) & $\leq 0.12-8$ & $\leq 0.12$ & 8 & \\
\hline & $\mathrm{FC} \mathrm{(5)}$ & $\leq 1$ & $\leq 1$ & $\leq 1$ & 100 \\
\hline & CAS (5) & $\leq 0.12-0.5$ & $\leq 0.12$ & 0.5 & \\
\hline & MIC (5) & $\leq 0.06-0.12$ & $\leq 0.06$ & 0.12 & \\
\hline \multirow{6}{*}{$\begin{array}{l}\text { Candida species non- } \\
\text { albicans ( } 3 \text { ) }\end{array}$} & FLU (3) & $2-8$ & 8 & 8 & 33.3 \\
\hline & AMB (3) & $0.5-\geq 16$ & 0.5 & $\geq 16$ & \\
\hline & VOR (2) & $\leq 0.12$ & $\leq 0.12$ & $\leq 0.12$ & \\
\hline & $\mathrm{FC} \mathrm{(3)}$ & $\leq 1$ & $\leq 1$ & $\leq 1$ & 100 \\
\hline & CAS (1) & 0.5 & 0.5 & 0.5 & \\
\hline & MIC (1) & 0.5 & 0.5 & 0.5 & \\
\hline \multirow[t]{6}{*}{ C. lipolytica (3) } & FLU (3) & $4-16$ & 8 & 16 & 0 \\
\hline & AMB (3) & $0.5-1$ & 0.5 & 1 & \\
\hline & VOR (3) & $\leq 0.12-0.25$ & $\leq 0.12$ & 0.25 & \\
\hline & $\mathrm{FC} \mathrm{(3)}$ & $4-8$ & 8 & 8 & 33.3 \\
\hline & CAS (2) & $\leq 0.12$ & $\leq 0.12$ & $\leq 0.12$ & \\
\hline & MIC & - & - & - & \\
\hline
\end{tabular}


Contd...

\begin{tabular}{|c|c|c|c|c|c|}
\hline Species (no. of isolates) & $\begin{array}{l}\text { Agents (no. available } \\
\text { for susceptibilities) }\end{array}$ & MIC $(\mu \mathrm{g} / \mathrm{mL})$ range & $M I C_{50}(\mu \mathrm{g} / \mathrm{mL})$ & $M I C_{90}(\mu g / m L)$ & Percentage sensitive \\
\hline \multirow[t]{6}{*}{ C. famata (3) } & FLU (3) & $\leq 0.5-4$ & $\leq 0.5$ & 4 & 66.7 \\
\hline & $\mathrm{AMB}(3)$ & $\leq 0.25$ & $\leq 0.25$ & $\leq 0.25$ & \\
\hline & $\operatorname{VOR}(2)$ & $\leq 0.12$ & $\leq 0.12$ & $\leq 0.12$ & \\
\hline & $\mathrm{FC}(3)$ & $\leq 1$ & $\leq 1$ & $\leq 1$ & 100 \\
\hline & CAS (1) & $\leq 0.12-0.25$ & 0.25 & 0.25 & \\
\hline & MIC (1) & $0.12-0.25$ & 0.12 & 0.25 & \\
\hline \multirow[t]{6}{*}{ C. glabrata (2) } & FLU (1) & 2 & 2 & 2 & 100 \\
\hline & $\mathrm{AMB}(2)$ & $0.5-1$ & 0.5 & 1 & 100 \\
\hline & $\operatorname{VOR}(1)$ & 0.25 & 0.25 & 0.25 & \\
\hline & $5-\mathrm{FC}(1)$ & $\leq 1$ & $\leq 1$ & $\leq 1$ & 100 \\
\hline & CAS (1) & $\leq 0.12$ & $\leq 0.12$ & $\leq 0.12$ & 100 \\
\hline & MIC (2) & $\leq 0.06$ & $\leq 0.06$ & $\leq 0.06$ & 100 \\
\hline \multirow[t]{6}{*}{ C. guilliermondii (2) } & FLU (2) & $2-4$ & 2 & 4 & 50 \\
\hline & $\operatorname{AMB}(2)$ & $\leq 0.25$ & $\leq 0.25$ & $\leq 0.25$ & 100 \\
\hline & VOR (2) & $\leq 0.12$ & $\leq 0.12$ & $\leq 0.12$ & \\
\hline & $\mathrm{FC}(1)$ & $\leq 1$ & $\leq 1$ & $\leq 1$ & 100 \\
\hline & CAS (1) & 0.5 & 0.5 & 0.5 & 100 \\
\hline & MIC (1) & 0.5 & 0.5 & 0.5 & 100 \\
\hline \multirow[t]{6}{*}{ C. Iusitaniae (2) } & FLU (2) & $\leq 1$ & $\leq 1$ & $\leq 1$ & 100 \\
\hline & $\mathrm{AMB}(2)$ & 0.5 & 0.5 & 0.5 & \\
\hline & $\operatorname{VOR}(2)$ & $\leq 0.12$ & $\leq 0.12$ & $\leq 0.12$ & \\
\hline & $\mathrm{FC}(2)$ & $\leq 1$ & $\leq 1$ & $\leq 1$ & 100 \\
\hline & CAS (1) & 1 & 1 & 1 & \\
\hline & MIC & - & - & - & \\
\hline \multirow[t]{6}{*}{ C. utilis (2) } & FLU (2) & $\leq 1-2$ & $\leq 1$ & 2 & 100 \\
\hline & $\mathrm{AMB}(2)$ & $\leq 0.25-0.5$ & $\leq 0.25$ & 0.5 & \\
\hline & VOR (2) & $\leq 0.12$ & $\leq 0.12$ & $\leq 0.12$ & \\
\hline & $\mathrm{FC} \mathrm{(2)}$ & $\leq 1$ & $\leq 1$ & $\leq 1$ & 100 \\
\hline & CAS (2) & $\leq 0.12$ & $\leq 0.12$ & $\leq 0.12$ & \\
\hline & MIC (2) & $\leq 0.06-0.12$ & $\leq 0.06$ & 0.12 & \\
\hline \multirow[t]{6}{*}{ Total (94) } & FLU (93) & $\leq 0.5-\geq 64$ & 1 & 8 & 71 \\
\hline & AMB (94) & $\leq 0.25-\geq 16$ & 0.5 & 1 & 91.5 \\
\hline & VOR (91) & $\leq 0.12-8$ & $\leq 0.12$ & 0.25 & 99 \\
\hline & FC (92) & $\leq 1-\geq 64$ & $\leq 1$ & 8 & 87.8 \\
\hline & CAS (56) & $\leq 0.12-1$ & $\leq 0.25$ & 0.5 & 94 \\
\hline & MIC (43) & $\leq 0.06-0.12$ & 0.5 & 0.12 & 100 \\
\hline
\end{tabular}

Flu: Fluconazole; AMB: Amphotericin B; VOR: Voriconazole; FC: Flucytosine; CAS: Caspofungin; MIC: Micafungin

underlying conditions. ${ }^{28}$ This is similar to our study with congenital malformation reported in $27 \%$ and hematological malignancy in $13.5 \%$ as the two predominant predisposing conditions. In our study, there were probably not enough patients with risk factors to draw any firm conclusion.

The overall mortality was found to be $31.3 \%$ in our study which is significantly higher than a study in Turkey (13.7\%) and Denmark (10.2\%). ${ }^{7,33}$ In another study, overall mortality was found to be $41 \% .{ }^{9}$ The highest mortality was seen in C. pelliculosa (23.3\%), followed by C. tropicalis (20\%), C. parapsilosis (16.7\%), C. albicans (10\%), and C. haemulonii (10\%). In another study in a PICU, C. albicans was found to have the highest mortality rate among all Candida species $(30.7 \%)^{7}$ In a Scottish study, mortality was primarily associated with C. albicans. (49\%), followed by C. glabrata (32\%), C. parapsilosis $(11.3 \%)$, and other species (7.7\%). ${ }^{9}$ When compared to adults, there was no apparent difference seen in mortality between C. albicans and non-C. albicans BSIs in our study which is similar to other reports in pediatric age-groups. ${ }^{32,34,35}$ Candidemia among children admitted in ICU was associated with higher mortality (33.8\%) in comparison to children admitted in wards (28.6\%). This finding is consistent with a review article on pediatric invasive candidiasis and another study among adults in a tertiary care Saudi hospital. ${ }^{3,10}$ In our study, mortality was found to be highest in 12 to 16-years agegroup ( $80 \%$ ) followed by in 2 to $<12$ years (39\%). This conclusion may not be clinically relevant as the total numbers in each category was less. In a nationwide cohort study from Denmark, the mortality was found to be highest for neonates (17.1\%). ${ }^{33}$

Most of the Candida spp. worldwide was found to be sensitive to polyene and echinocandins. ${ }^{12,13,36} \mathrm{C}$. parapsilosis remains $100 \%$ sensitive to all the tested antifungals in our study followed 
by C. albicans, $100 \%$ sensitive to all except flucytosine (92.3\%), C. tropicalis, $100 \%$ sensitive to all except fluconazole (95.5\%), and flucytosine (95.2\%). It was found that micafungin was the only antifungal that had $100 \%$ overall sensitivity to all Candida spp. followed by voriconazole $99 \%$. Our susceptibility data were similar to a surveillance study of candidemia in Scotland, with limited or no resistance found among C. albicans, C.parapsilosis, or C. tropicalis isolates. ${ }^{9}$ In the present study, the fluconazole sensitivity was $71 \%$ for Candida spp. The increase in fluconazole resistance is a matter of deep concern in disseminated candidiasis involving candidemia. ${ }^{21,37,38}$ This could be due to extensive use of fluconazole in various clinical conditions which also has led to dominance of non-C. albicans species over C. albicans. ${ }^{39}$ Clinicians should also keep in mind about intrinsic resistance of $C$. krusei isolates to fluconazole and decreased susceptibility to other antifungals. Hence speciation and susceptibility testing of all clinical isolates of candidemia is of utmost importance.

Only $75 \%$ of C. krusei were susceptible to caspofungin. This is similar to a study where they have reported $66.7 \%$ of C. krusei isolates susceptible to caspofungin, in contrast to another study where the C. krusei showed $100 \%$ sensitivity to caspofungin. ${ }^{12,21}$

\section{LIMITATIONS}

The findings in this report are subject to certain limitations. The underlying conditions and predisposing factors in our study were extracted from hospital information system, which might have resulted in underestimates of certain conditions, if not entered in the information system due to busy schedule of the healthcare workers. In addition, the underestimates of true proportion of invasive candidiasis could be due to difficulty in obtaining adequate blood sample volume as per the age of the children recommended in the automated culture bottles. It is a common practice among the clinicians to obtain only one blood culture bottle instead of multiple sets, probably to minimize the treatment cost to the patient. It is an established fact that the blood volume plays an important role in the optimal sensitivity of the automated blood culture system. The number of days before the episode of candidemia, antifungal treatment and length of stay in our study could not be evaluated.

\section{Conclusion}

Candidemia remains one of the serious causes of illness and death worldwide. Uncommon Candida species have emerged as causative agents of invasive fungal infections among children.

These pathogens frequently have shown decreased susceptibility to fluconazole, the most frequently used antifungals. Therefore, surveillance data for candidemia should continue to monitor incidence trends by age, species distribution, changes in underlying predisposing factors, track emergence of resistance, and assess trends in antifungal treatment. Finally, understanding the epidemiology of Candida spp. is critical as it is expected to improve the development of treatment, outcome and preventive efforts.

\section{References}

1. Toda M, Williams SR, Berkow EL, et al. Population-based active surveillance for culture-confirmed candidemia - four sites, United States, 2012-2016. MMWR Surveill Summ 2019;68(8):1-15. DOI: 10.15585/mmwr.ss6808a1.

2. Sievert DM, Ricks P, Edwards JR, et al. Antimicrobial-resistant pathogens associated with healthcare-associated infections: summary of data reported to the national healthcare safety network at the centers for disease control and prevention, 2009-2010. Infect Control Hosp Epidemiol 2013;34(1):1-14. DOI: 10.1086/668770.

3. Steinbach WJ. Pediatric invasive candidiasis: epidemiology and diagnosis in children. J Fungi (Basel) 2016;2(1):5. DOI: 10.3390/ jof2010005.

4. Kalkanci A, Dizbay M, Turan O, et al. Nosocomial transmission of Candida pelliculosa fungemia in a pediatric intensive care unit and review of the literature. Turk J Pediatr 2010;52(1):42-49.

5. Taylor G, Buchanan-Chell M, Kirkland T, et al. Long term trends in the occurrence of nosocomial blood stream infection. Can J Infect Dis 2000;11(1):29-33. DOI: 10.1155/2000/393194.

6. Pfaller MA, Diekema DJ. Epidemiology of invasive candidiasis: a persistent public health problem. Clin Microbiol Rev 2007;20(1):133163. DOI: 10.1128/CMR.00029-06.

7. Aslan N, Yildizdas D, Alabaz D, et al. Invasive Candida infections in a pediatric intensive care unit in Turkey: evaluation of an 11-year period. J Pediatr Intensive Care 2020;9(1):21-26. DOI: 10.1055/s-00391695061.

8. Dadar M, Tiwari R, Karthik K, et al. Candida albicans - biology, molecular characterization, pathogenicity, and advances in diagnosis and control - an update. Microb Pathog 2018;117:128-138. DOI: 10.1016/j.micpath.2018.02.028.

9. Rajendran R, Sherry L, Deshpande A, et al. A prospective surveillance study of candidaemia: epidemiology, risk factors, antifungal treatment and outcome in hospitalized patients. Front Microbiol 2016;7:915. DOI: 10.3389/fmicb.2016.00915.

10. Alkharashi N, Aljohani S, Layqah L, et al. Candida bloodstream infection: changing pattern of occurrence and antifungal susceptibility over 10 Years in a tertiary care Saudi hospital. Can J Infect Dis Med Microbiol 2019;2019:2015692. DOI: 10.1155/2019/2015692.

11. Puig-Asensio M, Ruiz-Camps I, Fernández-Ruiz M, et al. Epidemiology and outcome of candidaemia in patients with oncological and haematological malignancies: results from a population-based surveillance in Spain. Clin Microbiol Infect 2015;21(5). DOI: 10.1016/j. cmi.2014.12.027.

12. Omrani AS, Makkawy EA, Baig K, et al. Ten-year review of invasive Candida infections in a tertiary care center in Saudi Arabia. Saudi Med J 2014;35(8):821-826.

13. Lyon GM, Karatela S, Sunay S, et al. Candida surveillance study investigators. Antifungal susceptibility testing of Candida isolates from the Candida surveillance study. J Clin Microbiol 2010;48(4):12701275. DOI: 10.1128/JCM.02363-09.

14. Asmundsdottir LR, Erlendsdottir H, Gottfredsson M. Nationwide study of candidemia, antifungal use, and antifungal drug resistance in Iceland, 2000 to 2011. J Clin Microbiol 2013;51(3):841-848. DOI: 10.1128/JCM.02566-12.

15. Otag F, Yarpuzlu M, Gulbudak H, et al. Candida pelliculosa fungemia cases in pediatric intensive care unit. Pediatr Inf 2015;9(2):85-90. DOI: 10.5152/ced.2015.1662.

16. Chatterjee S, Alampalli SV, Nageshan RK, et al. Draft genome of a commonly misdiagnosed multidrug resistant pathogen Candida auris. BMC Genomics 2015;16(1):686. DOI: 10.1186/s12864-0151863-z.

17. Clinical and Laboratory Standards Institute. Reference Method for Broth Dilution Antifungal Susceptibility Testing of Yeasts-Third Edition: Approved Standard M27-A3. CLSI, Wayne, PA, USA, 2008.

18. The European Committee on Antimicrobial Susceptibility Testing. Breakpoint tables for interpretation of MICs for antifungal agents, version 10.0, 2020. http://www.eucast.org/astoffungi/ clinicalbreakpointsforantifungals/.

19. Jung $\mathrm{SI}$, Shin $\mathrm{JH}, \mathrm{Choi} \mathrm{HJ}$, et al. Antifungal susceptibility to amphotericin B, fluconazole, voriconazole, and flucytosine in Candida bloodstream isolates from 15 tertiary hospitals in Korea. Ann Lab Med 2012;32(6):426-428. DOI: 10.3343/alm.2012.32.6.426.

20. Wattal C, Goel N. Pediatric blood cultures and antibiotic resistance: an overview. Indian J Pediatr 2020;87(2):125-131. DOI: 10.1007/s12098019-03123-y. 
21. Kaur R, Jaggi S, Dhakad MS, et al. An etiological and antifungal profile of candidemia in children. Int J Community Med Public Health 2019;6(9):3899-3904. DOI: 10.18203/2394-6040.ijcmph20193990.

22. Tak V, Mathur $P$, Varghese $P$, et al. The epidemiological profile of candidemia at an Indian trauma care center. J Lab Physicians 2014;6(2):96-101. DOI: 10.4103/0974-2727.141506.

23. Gonzalez GM, Trevino-Rangel RJ, Palma-Nicolas JP, et al. Species distribution and antifungal susceptibility of bloodstream fungal isolates in paediatric patients in Mexico: a nationwide surveillance study. J Antimicrob Chemother 2013;68(12):2847-2851. DOI: 10.1093/ $\mathrm{jac} / \mathrm{dkt} 283$.

24. Singh R, Parija SC. Candida parapsilosis: an emerging fungal pathogen. Indian J Med Res 2012;136(4):671-673.

25. Warris A. Candida auris, what do paediatricians need to know? Arch Dis Child 2018;103(9):891-894. DOI: 10.1136/archdischild-2017-313960.

26. Rodrigues LS, Motta FA, Picharski GL, et al. Invasive candidiasis: risk factor for mortality in a pediatric tertiary care hospital in south of Brazil. Medicine (Baltimore) 2019;98(23):e15933. DOI: 10.1097/ MD.0000000000015933.

27. Charsizadeh A, Mirhendi H, Nikmanesh B, et al. Candidemia in children caused by uncommon species of Candida. Arch Pediatr Infect Dis 2018;6(2):e11895. DOI: 10.5812/pedinfect.11895.

28. Tragiannidis A, Fegeler W, Rellensmann G, et al. Candidaemia in a European paediatric university hospital: a 10-year observational study. Clin Microbiol Infect 2012;18(2):E27-E30. DOI: 10.1111/j.14690691.2011.03720.x.

29. Lin HC, Lin HY, Su BH, et al. Reporting an outbreak of Candida pelliculosa fungemia in a neonatal intensive care unit. J Microbiol Immunol Infect 2013;46(6):456-462. DOI: 10.1016/j.jmii.2012.07.013.

30. Krcmery V, Kisac P, Liskova A. Voriconazole and posaconazole resistant Candida pelliculosa fungemia after cardiac surgery. Pediatr Infect Dis J 2009;28(1):75-76. DOI: 10.1097/INF.0b013e31818edde1.
31. Santolaya ME, Thompson L, Benadof $D$, et al. A prospective, multicenter study of Candida bloodstream infections in Chile. PLoS ONE 2019;14(3):e0212924. DOI: 10.1371/journal.pone.0212924.

32. Dutta A, Palazzi DL. Candida non-albicans versus Candida albicans fungemia in the non-neonatal pediatric population. Pediatr Infect Dis J 2011;30(8):664-668. DOI: 10.1097/INF.0b013e318213da0f.

33. Lausch KR, Schultz Dungu KH, Callesen MT, et al. Pediatric candidemia epidemiology and morbidities: a nationwide cohort. Pediatr Infect Dis J 2019;38(5):464-469. DOI: 10.1097/INF.0000000000002207.

34. Pappas PG, Rex JH, Lee J, et al. A prospective observational study of candidemia: epidemiology, therapy, and influences on mortality in hospitalized adult and pediatric patients. Clin Infect Dis 2003;37(5):634-643. DOI: 10.1086/376906.

35. Wisplinghoff $\mathrm{H}, \mathrm{Bischoff} \mathrm{T}$, Tallent $\mathrm{SM}$, et al. Nosocomial bloodstream infections in US hospitals: analysis of 24,179 cases from a prospective nationwide surveillance study. Clin Infect Dis 2004;39(3):309-317. DOI: $10.1086 / 421946$.

36. Al-Dorzi HM, Sakkijha H, Khan R, et al. Invasive candidiasis in critically ill patients: a prospective cohort study in two tertiary care centers. J Intensive Care Med 2018(6):885066618767835. DOI: 10.1177/0885066618767835.

37. Adhikary R, Joshi S. Species distribution and anti-fungal susceptibility of Candidaemia at a multi super-specialty center in Southern India. Indian J Med Microbiol 2011;29(3):309-311. DOI: 10.4103/02550857.83920 .

38. Badiee P, Alborzi A. Susceptibility of clinical Candida species isolates to antifungal agents by E-test, Southern Iran: a five year study. Iran J Microbiol 2011;3(4):183-188.

39. Kothavade RJ, Kura MM, Valand AG, et al. Candida tropicalis: its prevalence, pathogenicity and increasing resistance to fluconazole. J Med Microbiol 2010;59(Pt 8):873-880. DOI: 10.1099/jmm 0.013227-0. 